\title{
Extreme regimes of femtosecond photoemission from a copper cathode in a dc electron gun
}

\author{
P. L. E. M. Pasmans, ${ }^{1}$ D. C. van Vugt, ${ }^{1}$ J. P. van Lieshout, ${ }^{1}$ G. J. H. Brussaard, ${ }^{1}$ and O. J. Luiten ${ }^{1,2, *}$ \\ ${ }^{1}$ Department of Applied Physics, Eindhoven University of Technology, \\ P.O. Box 13, 5600 MB Eindhoven, The Netherlands \\ ${ }^{2}$ Institute of Complex Molecular Systems, Eindhoven University of Technology, \\ P.O. Box 13, 5600 MB Eindhoven, The Netherlands
}

(Received 18 August 2016; published 26 October 2016)

\begin{abstract}
The femtosecond photoemission yield from a copper cathode and the emittance of the created electron beams has been studied in a $12 \mathrm{MeV} / \mathrm{m}, 100 \mathrm{keV}$ dc electron gun over a wide range of laser fluence, from the linear photoemission regime until the onset of image charge limitations and cathode damaging. The measured photoemission curves can be described well with available theory which includes the Schottky effect, second-order photoemission, and image charge limitation. The second-order photoemission can be explained by thermally assisted one-photon photoemission (1PPE) and by above-threshold two-photon photoemission (2PPE). Measurements with a fresh cathode suggest that the 2PPE process is dominant. The beam emittance has been measured for the entire range of initial surface charge densities as well. The emittance measurements of space-charge dominated beams can be described well by an envelope equation with generalized perveance. The dc gun produces $0.1 \mathrm{pC}$ bunches with $25 \mathrm{~nm}$ rms normalized emittance, corresponding to a normalized brightness usually associated with rf photoguns. In this experimental study the limits of femtosecond photoemission from a copper cathode have been explored and analyzed in great detail, resulting in improved understanding of the underlying mechanisms.
\end{abstract}

DOI: 10.1103/PhysRevAccelBeams.19.103403

\section{INTRODUCTION}

Photoemission electron sources play an essential role in many experiments aimed at the study of ultrafast structural dynamics [1-5]. After creation through photoemission with femtosecond laser pulses, ultrashort electron bunches can be used for electron diffraction and imaging or to enable $\mathrm{x}$-ray diffraction experiments with a free-electron laser (FEL). With ultrafast electron diffraction (UED), ultrafast electron microscopy (UEM), or ultrafast x-ray diffraction, transformations of atomic structures can be captured with both atomic spatial resolution and atomic temporal resolution [6-14]. These techniques provide a deeper, fundamental understanding of atomic dynamics in matter ranging from solid-state materials like graphene and superconductors to complex biological structures such as proteins and viruses [10-14].

For x-ray FELs, electron bunches are typically created by photoemission in radio-frequency (rf) photoguns and subsequently accelerated in $\mathrm{rf}$ linacs to $\mathrm{GeV}$ energies to generate $\mathrm{x}$-rays in an undulator. In a recent development the few-MeV pulsed electron beams produced with rf

\footnotetext{
o.j.luiten@tue.nl

Published by the American Physical Society under the terms of the Creative Commons Attribution 3.0 License. Further distribution of this work must maintain attribution to the author $(s)$ and the published article's title, journal citation, and DOI.
}

photoguns are applied for electron diffraction, which is a particularly promising approach for attaining ultrashort electron bunches and for gas-phase samples $[15,16]$. For most applications of electron diffraction and imaging, however, electron energies are preferred in the range of $30-200 \mathrm{keV}$ because of the relatively high elastic scattering cross-section at these energies, the relatively low radiation damage, and the ability to accelerate with stable static fields in a dc photogun [4].

Both metallic and semiconductor photocathodes can be used $[1,2]$. Semiconductor cathodes typically have a higher yield, but metallic cathodes are generally more stable, have less stringent vacuum requirements, and exhibit prompt emission of the electrons. The latter is particularly relevant if sub-ps temporal resolution is required in a compact setup.

Photoemission has been used and studied extensively for many decades in the context of both photoelectron spectroscopy and photoemission electron sources [17-26]. Most photoemission studies are performed in the low field regime with spectroscopy or in high rf fields for generation of relativistic beams. The application of femtosecond photoemission in a high-field-strength dc gun for UED is a relatively recent development and the ultimate limits of photoemission for UED still need to be explored in detail. The investigation of the different regimes of photoemission is particularly important for single-shot electron diffraction, which requires the generation of as much charge as possible from an illumination area as small as possible. 
Here, we report on photoemission yield and emittance measurements in the $12 \mathrm{MeV} / \mathrm{m}, 100 \mathrm{keV}$ dc electron gun developed for the UED setup at Eindhoven University of Technology. At the time of the experiments, the copper cathode had been in use for 9 months and was not cleaned prior to the measurements. This represents the typical situation when the electron gun is used for UED experiments. The photoemission yield has been studied over a wide range of laser fluence, ranging from the regime of linear photoemission, where a few electrons per $\mu \mathrm{m}^{2}$ are extracted from the photocathode, until the onset of image charge limitations and cathode damaging, where $10^{3}-10^{4}$ electrons per $\mu \mathrm{m}^{2}$ are extracted, comparable to the surface charge densities in rf photoguns. The whole fluence range has been measured in the same setup with a single copper cathode illuminated with femtosecond UV laser pulses with a central wavelength of $267 \mathrm{~nm}$. The acceleration field has been varied between $1.23 \mathrm{MV} / \mathrm{m}$ and $12.3 \mathrm{MV} / \mathrm{m}$, corresponding to electron energies of $10 \mathrm{keV}$ to $100 \mathrm{keV}$. Emittance measurements have been performed using waist scans for initial surface charge densities ranging from 10 to $10^{3}$ electrons per $\mu \mathrm{m}^{2}$ and for an acceleration field of $12.3 \mathrm{MV} / \mathrm{m}$ in order to study the influence of the charge density on beam quality.

In Sec. II, the theoretical framework is presented to describe the femtosecond photoemission for the complete range of fluences and acceleration fields. This includes the Schottky effect, nonlinear photoemission, and image charge limitation. The model for image charge limited photoemission is compared with particle tracking simulations using the general particle tracer (GPT) code. In Sec. II E the theoretical background of beam emittance and brightness is discussed and how to model the emittance evolution of space-chargedominated beams. The experimental setup for the photoemission yield and emittance measurements is described in Sec. III and the results are presented and discussed in Sec. IV. We find that both the photoemission yield measurements and the measured evolution of the beam emittance agree very well with theoretical model descriptions. For a proper description of the photoemission process it is essential that the Schottky effect, second-order photoemission, and image charge limitation are included. In particular second-order photoemission turns out to play an important role under all conditions relevant for UED or UEM. Measurements with a fresh cathode suggest that abovethreshold two-photon emission (2PPE) is the dominant mechanism. The emittance measurements of space-charge dominated beams can be described very well using an envelope equation with generalized perveance. Without any particular efforts to minimize space-charge effects by optimizing the photoemission beam profile, the dc gun produces $0.1 \mathrm{pC}$ bunches - sufficient for single-shot UEDwith $25 \mathrm{~nm}$ rms normalized emittance from a $25 \mu \mathrm{m} \mathrm{rms}$ spot size. This corresponds to a normalized brightness usually associated with rf photoguns.

\section{ULTRAFAST PHOTOEMISSION THEORY}

\section{A. Work function and Schottky effect}

Photoemission is determined by the (surface) work function $\phi$ of a material, which is the minimum energy required to remove an electron at the Fermi level from a solid into vacuum. The reported work function of copper (in zero field) lies in the range $\phi_{0}=4.31-4.91 \mathrm{eV}$, depending on the orientation of the lattice planes and on the cleaning of the cathode [27,28]. When an external field is applied, the effective work function $\phi$ is lowered due to the Schottky effect [29]:

$$
\phi=\phi_{0}-\sqrt{\frac{e^{3} \beta G_{a}}{4 \pi \epsilon_{0}}}
$$

where $G_{a}$ is the applied acceleration field, $e$ is the electron charge, $\epsilon_{0}$ is the vacuum permittivity, and $\beta$ is the field enhancement factor due to surface roughness ( $\beta=1$ for a perfectly flat cathode). Literature values for $\beta$ range from 1.5 to $500[23,26]$. The work function is lowered by an amount $\Delta \phi=0.12 \mathrm{eV}$ for $G_{a}=$ $10 \mathrm{MV} / \mathrm{m}$ and $\beta=1$.

\section{B. Generalized Fowler-DuBridge theory}

We apply the generalized Fowler-DuBridge theory [17-19], which is sufficient for our purposes. More advanced theories have recently been developed, such as [30], but these are not relevant to the bare copper emission surface in our setup. Following the generalized FowlerDuBridge theory the photoemitted current density $J$ can be written as the sum of the partial current densities $J_{n}$ given by:

$$
J_{n}(\boldsymbol{r}, t)=a_{n}\left[\frac{e}{h \nu}(1-R) I(\boldsymbol{r}, t)\right]^{n} A_{0} T_{e}^{2} f\left(x_{n}\right)
$$

with

$$
\begin{gathered}
f\left(x_{n}\right)=\int_{0}^{\infty} \ln \left[1+\exp \left(-y+x_{n}\right)\right] \mathrm{d} y \\
x_{n}=\frac{n h \nu-\phi}{k_{B} T_{e}}
\end{gathered}
$$

where $a_{n}$ are phenomenological coefficients, $h$ is Planck's constant, $h \nu$ is the incident photon energy, $R$ is the surface reflectivity, $I$ is the incident (laser) irradiance, $A_{0}=1.20173 \times 10^{6} \mathrm{~A} / \mathrm{m}^{2} \mathrm{~K}^{2}$ is the Richardson constant, $T_{e}$ is the electron surface temperature, $k_{B}$ is Boltzmann's constant, and $f\left(x_{n}\right)$ is the Fowler function [17]. The term $a_{n}[\ldots]^{n}$ in Eq. (2) is proportional to the probability per unit time of an electron to absorb $n$ photons and to escape [21]. The term $T_{e}^{2} f\left(x_{n}\right)$ in Eq. (2) takes into 
account the number of available electrons at the temperature $T_{e}$ (based on Fermi-Dirac statistics) to overcome the work function of the metal and describes thermally assisted photoemission. For $n=0$, the partial current reduces to the Richardson equation for thermionic emission, $J_{0}=a_{0} A_{0} T_{e}^{2} \exp \left(-\phi / k_{B} T_{e}\right)$, with $a_{0}$ a dimensionless constant of order unity [31]. For $n \neq 0, J_{n}$ describes $n$-photon photoemission with constants $a_{n}$ which can be assessed experimentally or evaluated by microscopic theories. The ratio between the constants can be estimated roughly using the scaling relation [21]: $a_{n} / a_{n+1} \sim 10^{15}-10^{18} \mathrm{~A} / \mathrm{m}^{2}$. In Sec. II C it will be shown that for $h \nu-\phi \gg k_{B} T_{e}$ the constant $a_{1}$ can be related to the (linear) quantum efficiency according to $Q E \simeq a_{1} A_{0}(h \nu-\phi)^{2} / 2 k_{B}^{2}$. For copper $a_{1}=5 \times 10^{-18} \mathrm{~m}^{2} / \mathrm{A}$, as calculated using literature values from [29].

\section{Femtosecond photoemission}

For photoemission with sub-picosecond laser pulses, the thermodynamic equilibrium between the electron gas and the metal lattice is disturbed during the entire interaction time with the laser pulse [21,22]. The nonequilibrium regime is commonly described by the two-temperature model, in which the electrons and lattice are described by two separated systems with an electron temperature $T_{e}$ and a lattice temperature $T_{l}$. Equilibration between electrons and lattice is governed by the specific heat capacity of the electrons $C_{e}=\kappa T_{e}$ and the electron-phonon coupling parameter $g$. The typical rate for energy exchange $g / \kappa$ is in the order of $1 \mathrm{~K} / \mathrm{fs}$ for most metals, implying a time to equilibrium of the order of 1 ps [22]. During the $\sim 100 \mathrm{fs}$ photoemision process the electron temperature $T_{e}$ may therefore increase significantly with laser intensity $I(\boldsymbol{r}, t)$. Inspection of the general expression (2) shows that this implies an increase of the thermionic emission $J_{0}$. In addition it will result in thermally assisted nonlinear photoemission $J_{n}$ with a power dependence on $I$ greater than $n$, in particular thermally assisted second order photoemission for $n=1$. Equation (2) can be simplified by using the approximation $f(x) \simeq x^{2} / 2+\pi^{2} / 6$ for the Fowler function, which holds for $x>1$. For $n=1$ we then find:

$$
\begin{aligned}
J_{1}(\boldsymbol{r}, t) \simeq & \frac{a_{1} A_{0} e}{2 h \nu}(1-R) I(\boldsymbol{r}, t) \\
& \times\left[\left(\frac{h \nu-\phi}{k_{B}}\right)^{2}+\frac{\pi^{2} T_{e}(\boldsymbol{r}, t)^{2}}{3}\right] .
\end{aligned}
$$

For $h \nu-\phi \gg k_{B} T_{e}$, i.e., $x_{1} \gg 1, J_{1}$ becomes independent of $T_{e}$, resulting in the simple relation between $a_{1}$ and the linear QE mentioned in Sec. II B. Generally, $x_{n}=$ $n h \nu-\phi \gg k_{B} T_{e}$ for $n \geq 2$ so then the Fowler function may be approximated by $f(x) \simeq x^{2} / 2$, resulting in:

$$
J_{2}(\boldsymbol{r}, t) \simeq \frac{a_{2} A_{0} e^{2}}{2(h \nu)^{2}}[(1-R) I(\boldsymbol{r}, t)]^{2}\left(\frac{2 h \nu-\phi}{k_{B}}\right)^{2} .
$$

Assuming that equilibration of the electrons with the lattice takes place on time scales much longer than the laser pulse duration and that the laser pulse has a Gaussian temporal intensity profile with rms pulse length $\tau$, the final electron temperature in the photoemission process is given by [22]

$$
T_{e, \text { peak }}(\boldsymbol{r})=\sqrt{\frac{2 \alpha}{\kappa} F_{a}(\boldsymbol{r})+T_{i}^{2}}
$$

where $T_{i}$ is the initial temperature, $\alpha$ the optical absorption coefficient, and $F_{a}(\boldsymbol{r}) \equiv(1-R) \sqrt{2 \pi} \tau I_{0}(\boldsymbol{r})$ is the absorbed fluence with $I_{0}(\boldsymbol{r})$ the peak laser intensity as a function of position. For copper $\alpha=7.9 \times 10^{7} \mathrm{~m}^{-1}$ for $267 \mathrm{~nm}$ [32,33] and $\kappa=96.8 \mathrm{~J} / \mathrm{m}^{3} \mathrm{~K}^{2}$ [34], which gives $\alpha / \kappa=8 \times 10^{5} \mathrm{~m}^{2} \mathrm{~K}^{2} / \mathrm{J}$.

Integration of $J_{1}(\boldsymbol{r}, t)$ and $J_{2}(\boldsymbol{r}, t)$ over times $t \gg \tau$ and using Eq. (6) now yields the final (surface) charge densities $\sigma_{1}(\boldsymbol{r})$ and $\sigma_{2}(\boldsymbol{r})$ :

$$
\begin{gathered}
\sigma_{1}(\boldsymbol{r}) \simeq \frac{a_{1} A_{0} e}{2 h \nu}\left(\frac{h \nu-\phi}{k_{B}}\right)^{2} F_{a}(\boldsymbol{r}) \\
+\frac{a_{1} A_{0} e \pi^{2} \alpha}{6 h \nu \kappa} F_{a}(\boldsymbol{r})^{2} \\
\sigma_{2}(\boldsymbol{r}) \simeq \frac{a_{2} A_{0} e^{2}}{4 \tau \sqrt{\pi}(h \nu)^{2}}\left(\frac{2 h \nu-\phi}{k_{B}}\right)^{2} F_{a}(\boldsymbol{r})^{2} .
\end{gathered}
$$

The contribution of $T_{i}$ has been neglected since generally $k_{B} T_{i} \ll h \nu-\phi$. The second-order term for $\sigma_{1}$ appears due to the dependence of $T_{e}^{2}$ on $I_{0}$ (or $F_{a}$ ).

Equations (7) and (8) show that there are two different mechanisms which can explain a second order dependence of the photoemission yield on the laser fluence. The first mechanism is called thermally assisted one-photon photoemission (1PPE), expressed by the last term in Eq. (7). The second mechanism is called above-threshold twophoton photoemission (2PPE), expressed by Eq. (8). Above-threshold means that more photons are absorbed than the minimal number required to overcome the work function potential [22]. To estimate the relative contributions, the constants $b_{1}$ and $b_{2}$ are introduced, defined by: $\sigma(\boldsymbol{r})=\sigma_{1}(\boldsymbol{r})+\sigma_{2}(\boldsymbol{r}) \equiv b_{1} F_{a}(\boldsymbol{r})+b_{2} F_{a}(\boldsymbol{r})^{2}$. Using $a_{1} \simeq 5 \times 10^{-18} \mathrm{~m}^{2} / \mathrm{A}$ and $a_{2} \simeq 5 \times 10^{-33} \mathrm{~m}^{4} / \mathrm{A}^{2}$ from Sec. II B, with the parameters $\alpha / \kappa=8 \times 10^{5} \mathrm{~m}^{2} \mathrm{~K}^{2} / \mathrm{J}$, $h \nu=4.65 \mathrm{eV}, \phi=4.3 \mathrm{eV}$, and $\tau=100 \mathrm{fs}$, we find $b_{1}=1.1 \times 10^{-5} \mathrm{C} / \mathrm{J}$. Assuming thermally assisted $1 \mathrm{PPE}$ is the dominant second-order mechanism it follows that $b_{2}=1.7 \times 10^{-6} \mathrm{Cm}^{2} / \mathrm{J}^{2}$; if above-threshold $2 \mathrm{PPE}$ is dominant then $b_{2}=1.3 \times 10^{-6} \mathrm{Cm}^{2} / \mathrm{J}^{2}$. Both mechanisms could therefore contribute approximately equally to $b_{2}$. 


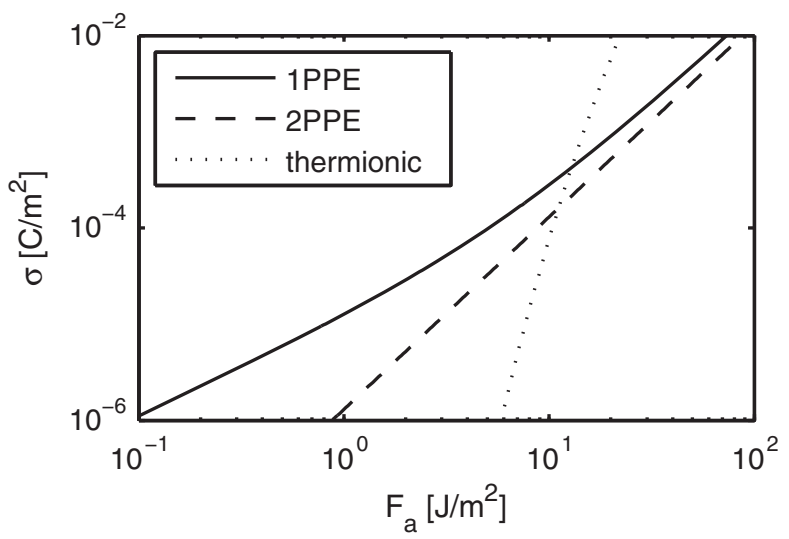

FIG. 1. One-photon photoemission (1PPE) and two-photon photoemission (2PPE) compared with thermionic emission as a function of absorbed fluence.

Obviously, the increase of $T_{e}$ also enhances the thermionic emission current density $J_{0}$, see Eq. (2). In Fig. 1, the surface charge density $\sigma$ is plotted as a function of the absorbed fluence $F_{a}$, for thermionic emission $\left(J_{0}\right)$, singlephoton photoemission $\left(J_{1}\right)$, and two-photon photoemission $\left(J_{2}\right)$, calculated using the parameters given above and using $T_{i}=300 \mathrm{~K}$ and $a_{0}=1$. To calculate the surface charge density $\sigma$ from $J_{0}$, constant thermionic emission during 1 ps has been assumed. Figure 1 shows that, for these parameters, thermionic emission becomes significant for $F_{a}>10 \mathrm{~J} / \mathrm{m}^{2}$. The curves for $1 \mathrm{PPE}$ and $2 \mathrm{PPE}$ obtain the same slope for high values of $F_{a}$; whether these curves cross depends on the variables $h \nu, \tau$, and $\phi\left(G_{a}\right)$, and on the constants $a_{1}, \alpha / \kappa$, and $a_{2}$.

\section{Image charge limited emission}

With increasing laser fluence and, consequently, an increasing photoemitted surface charge density, the photoemission yield eventually becomes suppressed due to image charge. The most elegant way to take the effect of the image charge into account is by including the image charge into the potential barrier for the photoemission process [35]. Here, we apply a simpler model in which we treat the photoemitted electrons as a single sheet which shields the cathode from the acceleration field. The surface charge density threshold $\sigma_{\lim }$ at which the field at the cathode is cancelled is then easily derived analytically [36]: $\sigma_{\lim } \equiv \epsilon_{0} \beta G_{a}$. For $\beta=1$ and $G_{a}=10 \mathrm{MV} / \mathrm{m}$, $\sigma_{\lim }=8.9 \times 10^{-5} \mathrm{C} / \mathrm{m}^{2}$.

For the spatial profile of the laser pulse, a cylindrically symmetric Gaussian distribution is assumed with an $\mathrm{rms}$ width $s$. This gives a peak fluence $F_{0} \equiv E / 2 \pi s^{2}$ for a pulse energy $E$. The image charge limitation can be included by assuming that the photoemitted surface charge density $\sigma(\boldsymbol{r})$ is locally limited by $\sigma_{\lim }$. The fluence $F_{\text {lim }}$ is defined as the fluence at which $\sigma(\boldsymbol{r})=\sigma_{\mathrm{lim}}$. Integration of $\sigma(\boldsymbol{r})$ over the Gaussian laser profile gives:

$$
\begin{aligned}
\sigma_{0} & \equiv \frac{Q}{2 \pi s^{2}} \\
& = \begin{cases}b_{1} F_{0}+\frac{b_{2}}{2} F_{0}^{2} & \text { for } F_{0} \leq F_{\lim } \\
b_{1} F_{\lim }+\frac{b_{2}}{2} F_{\lim }^{2}+\sigma_{\lim } \ln \left(\frac{F_{0}}{F_{\lim }}\right) & \text { for } F_{0} \geq F_{\text {lim }} .\end{cases}
\end{aligned}
$$

Note that for $F_{0} \leq F_{\text {lim }}, \sigma_{0}$ is the peak surface charge density; for $F_{0} \geq F_{\lim }, \sigma_{0}\left(\neq \sigma_{\lim }\right)$ becomes an "effective" peak surface charge density. Using $b_{1}$ and $b_{2}$ for 1PPE from the previous section gives $F_{\lim }=4.7 \mathrm{~J} / \mathrm{m}^{2}$ for $\beta=1$ and $G_{a}=10 \mathrm{MV} / \mathrm{m}$. Note that $F_{\lim }<10 \mathrm{~J} / \mathrm{m}^{2}$ for the chosen parameters, so that thermionic emission can safely be neglected below the onset of image charge limited emission.

In Fig. 2, Eq. (9) is compared with particle tracking simulations to check the validity of the model, using the general particle tracer (GPT) code [37]. The model is based on a homogeneous sheet of electrons, while the simulations are performed with electrons distributed according to a randomized Gaussian distribution in three dimensions. For these simulations, we created an electron distribution with $10^{6}$ macroparticles, with a temporal width of $\tau=100 \mathrm{fs}$, and with a beam temperature of $k_{B} T_{b}=0.5 \mathrm{eV}$. Spatially, the cylindrically symmetric distribution is split in a part for first-order photoemission with a spatial width $s_{1}=30 \mu \mathrm{m}$ and first-order coefficient $b_{1}=1 \times 10^{-5} \mathrm{C} / \mathrm{J}$, and in a part for second-order photoemission with a spatial width $s_{2}=s_{1} / \sqrt{2}$ and second-order coefficient $b_{2}=2 \times 10^{-6} \mathrm{Cm}^{2} / \mathrm{J}^{2}$. The parameters $b_{1}$ and $b_{2}$ also determine the amount of initially created electron charge. The electron distribution is created at $z=0.1 \mathrm{~nm}$ in a global acceleration field $G_{a}=10 \mathrm{MV} / \mathrm{m}$ which accelerates the electrons in the positive $z$ direction. Space charge and image charge effects are taken into account. Electrons which cross the $x y$-plane at $z=0$ are removed and electrons which cross the $x y$-plane at $z=1 \mathrm{~mm}$ are

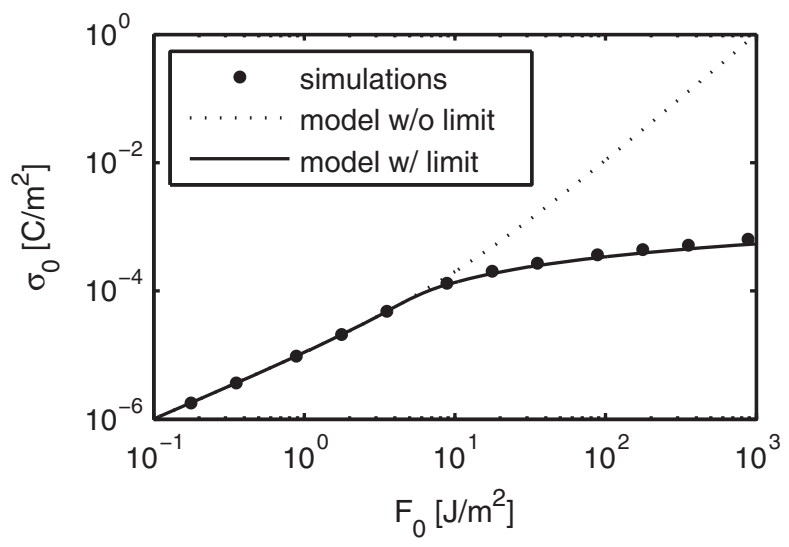

FIG. 2. Peak surface charge density versus peak laser fluence, simulated using GPT. 
counted as electrons that are photoemitted. Figure 2 shows that the model and simulations give the same result.

\section{E. Emittance and brightness}

The photoemission process influences the beam quality of the emitted electron bunches as expressed by emittance and brightness. Emittance is a measure for the focusability of a beam and is proportional to the area of the $2 \mathrm{D}$ projection of a bunch on $\left(x, p_{x}\right)$ phase space. The normalized (rms) emittance is defined as:

$$
\epsilon_{n, x}=\frac{1}{m c} \sqrt{\left\langle x^{2}\right\rangle\left\langle p_{x}^{2}\right\rangle-\left\langle x p_{x}\right\rangle^{2}}
$$

where $m$ is the electron mass, $c$ is the speed of light, and \langle\rangle indicates averaging over the electron distribution in the bunch. The emittances $\epsilon_{n, y}$ and $\epsilon_{n, z}$ are defined analogously. Geometrical emittance $\epsilon_{x}$ is proportional to the area in trace space $\left(x, x^{\prime}\right)$ and is related to the normalized emittance according to $\epsilon_{n, x} \equiv \gamma \beta_{f} \epsilon_{x}$ (where $\gamma$ is the Lorentz factor and $\beta_{f}$ the bunch speed normalized to the speed of light). In the fields of accelerator physics and electron microscopy, brightness is commonly used as a measure for beam quality and includes the bunch charge $Q$ as well. The normalized transverse brightness $B_{n}$ is defined as [38]:

$$
B_{n} \equiv \frac{1}{m c} \frac{Q}{(2 \pi)^{2} \epsilon_{n, x}^{2}} .
$$

The initial, or thermal, emittance is given by:

$$
\epsilon_{n, T}=s \sqrt{\frac{k_{B} T_{b}}{m c^{2}}}
$$

where $s$ is the rms laser spot size on the cathode and $T_{b}$ the effective source temperature. The source temperature is related to the difference between the photon energy and effective work function: $k_{B} T_{b} \simeq(h \nu-\phi) / 3$ [29]. For $h \nu=4.65 \mathrm{eV}, G_{a}=10 \mathrm{MV} / \mathrm{m}, \beta=1$, and $\phi_{0}=$ $4.31 \mathrm{eV}$, we have $k_{B} T_{b} \simeq 0.15 \mathrm{eV}$. Due to surface roughness, adsorbed compounds, the spectral width of (femtosecond) laser pulses, and other deviations from ideal photoemission, the beam temperature is higher in practice. The experimental thermal emittance is typically a factor 2-3 higher than the theoretical value [29]. In our calculations and simulations we assume $k_{B} T_{b}=0.5 \mathrm{eV}$.

The transverse emittance can be measured through a waist scan. The principle of a waist scan is to measure the rms electron beam size $s_{x}$ as a function of lens strength at a fixed position after the (electron-optical) lens. In the approximation that the bunch length is much larger than the bunch diameter, the propagation including space charge can be described to a reasonable approximation with the following single differential equation for the beam envelope [39]:

$$
s_{x}^{\prime \prime}-\frac{\epsilon_{x}^{2}}{s_{x}^{3}}-\frac{K}{s_{x}}=0
$$

where space charge is included by the term $-K / s_{x}$. Here $K$ is the generalized perveance which is proportional to the beam current. For a continuous $100 \mathrm{keV}$ electron beam with a top-hat profile and current $I_{b}$, the generalized perveance is given by $K=I_{b} / I_{P}$ with $I_{P} \simeq 2.4 \times 10^{3} \mathrm{~A}$ [39].

\section{EXPERIMENTAL SETUP}

The experiments have been performed in the UED setup at Eindhoven University of Technology as described in $[3,40-42]$.

\section{A. Laser fluence}

To generate ultrashort laser pulses, a Ti:sapphire oscillator (Coherent, Mantis) and a Ti:sapphire regenerative amplifier (Coherent, Legend Elite) are used. The amplified laser pulses have a central wavelength of $800 \mathrm{~nm}$ $(h \nu=1.55 \mathrm{eV})$ with a typical bandwidth of $32 \mathrm{~nm}$ (FWHM) and with a typical pulse length of $60 \mathrm{fs}$ (FWHM). UV light pulses with a central wavelength of $267 \mathrm{~nm}(h \nu=4.65 \mathrm{eV})$ are generated through third harmonic generation (THG) using thin BBO crystals. With a $\lambda / 2$-waveplate in a motorized rotation mount before the THG crystals, the energy of the UV pulses can be varied between $0.1 \mu \mathrm{J}$ and $4 \mu \mathrm{J}$. The UV pulses are focused onto the cathode in the electron gun, using a lens with a focal length of $f=1 \mathrm{~m}$. By changing the distance between this lens and the cathode or by removing the lens, the spot size $s$ on the cathode can be varied (see Table I). The spot size $s$ and energy per pulse $E$ are measured using a beam splitter between the lens and the cathode. The spot size $s$ is determined using a UV camera at the same distance behind the lens as the cathode. The camera image is subsequently fitted with a two-dimensional cylindrically symmetric Gaussian profile. The energy per pulse $E$ is determined with a UV diode (EPIGAP, EPD-440-0/1.45) which has been calibrated using a power meter (Coherent, LabMax Top with J-10MT-10kHz sensor). The signal of the UV diode is recorded with a digital oscilloscope (Agilent, DSO7054A). To calculate the absorbed energy $E_{a}$, the (measured) $85 \%$ transmission of the vacuum window (between lens and cathode) and the (theoretical) $34 \%$ reflectivity of the copper cathode are taken into account [32,33]. The

TABLE I. UV spot size for different lens positions with $f=1 \mathrm{~m}$.

\begin{tabular}{lr}
\hline \hline Lens position & \multicolumn{1}{c}{$s[\mu \mathrm{m}]$} \\
\hline No lens & $503.5 \pm 2.8$ \\
Lens @ $0.800 \mathrm{~m}$ & $168.7 \pm 1.4$ \\
Lens @ $0.950 \mathrm{~m}$ & $39.9 \pm 0.1$ \\
Lens@ $0.975 \mathrm{~m}$ & $32.8 \pm 0.7$ \\
\hline \hline
\end{tabular}


absorbed peak fluence is given by $F_{0}=E_{a} / 2 \pi s^{2}$. The angle of incidence of the UV pulses onto the cathode is $\approx 3^{\circ}$ with respect to normal incidence, so polarization effects are negligible.

\section{B. Charge density}

The photocathode material is high-purity (99.99\%), oxygen-free copper (Outokumpu, ASTM C10100), which was machined using single-diamond turning. The measurements have been performed with a cathode that had been in use for 9 months, except for a single measurement curve, which has been measured with a fresh cathode (as will be discussed in Sec. IV E). The cathode has a flat circular area in the center with a diameter of $1 \mathrm{~mm}$. The grooves from the diamond turning are used for alignment of the UV pulses onto the center of the cathode. The center of the grounded anode is at $11.4 \mathrm{~mm}$ from the cathode and has a circular opening with a $8.0 \mathrm{~mm}$ radius [41]. A maximal voltage difference of $100 \mathrm{kV}$ can be applied, which corresponds to an acceleration field of $-12.3 \mathrm{MV} / \mathrm{m}$ at the cathode, as calculated with the Poisson solver SUPERFISH. During the photoemission yield measurements, the voltage was varied from $-100 \mathrm{kV}$ to $-10 \mathrm{kV}$ in steps of $10 \mathrm{kV}$.

A magnetic coil is positioned after the photogun to focus the photoemitted electrons onto a Faraday cup. The current through the coil is optimized for each acceleration voltage to collect the maximal amount of charge. The Faraday cup has an opening diameter of $32 \mathrm{~mm}$ with a rather long core of $96 \mathrm{~mm}$, to prevent secondary electrons from escaping [43]. The Faraday cup is connected to a charge amplifier (Canberra, 2004) which is connected to the digital oscilloscope (Agilent, DSO7054A). The scope trace is used to determine the collected charge $Q$. The combination of $Q$ and the spot size $s$ from the UV camera is used to calculate the (effective) peak charge density $\sigma_{0}=Q / 2 \pi s^{2}$.

\section{Data aquisition}

The digital oscilloscope is connected to a computer which reads out the peak value of the trace from the UV diode and the complete trace from the charge amplifier. The trace from the charge amplifier has been fitted with an exponentially decaying step function in Matlab. The photoemission yield measurements have been performed for 10 voltages, 4 lens positions, and 360 steps of $\sim 1^{\circ}$ for the rotation mount (to vary the intensity). With 5 acquisitions per settings, this gives a total number of 72000 data points. The actual number of data points is somewhat less, since data points with $Q>12 \mathrm{pC}$ (clipping in charge amplifier) and with $E<100 \mathrm{~nJ}$ (noise level) have been discarded.

\section{Waist scans}

Waist scans to determine transverse bunch emittance have been performed using two magnetic coils. A first coil (as used for charge collection onto a Faraday cup) at $58 \mathrm{~mm}$ and a second coil at $320 \mathrm{~mm}$ behind the cathode. The first coil has an inner radius of $30.0 \mathrm{~mm}$, an outer radius of $51.0 \mathrm{~mm}$, a length $51.7 \mathrm{~mm}$ and 1055 windings. The second coil has an inner radius of $30.0 \mathrm{~mm}$, an outer radius of $45.9 \mathrm{~mm}$, a length $38.3 \mathrm{~mm}$ and 859 windings. The second coil is used to change the focusing strength, with the first condenser lens at a constant current of $I_{C 1}=2.91 \mathrm{~A}$. The electron bunches are recorded as function of the current $I_{C 2}$ through the second coil on a detector at a distance of $1.7 \mathrm{~m}$ from the cathode. The detector consists of a microchannel plate with a phospor screen (Burle Industries, APD 6040FM 12/10/8 I 60:1 P20) and a monochrome 10.7 megapixel camera (Lumenera, Lw11057M1). The bunches have been created from a laser spot on the cathode with an rms size $s=25.0 \pm 0.2 \mu \mathrm{m}$. For more details on the experimental setup, see Ref. [42].

\section{RESULTS AND DISCUSSION}

\section{A. Photoemission yield}

In Fig. 3 measurements are presented of the peak surface charge density $\sigma_{0} \equiv Q /\left(2 \pi s^{2}\right)$ (see Sec. II D) as a function of peak fluence $F_{0}$ for acceleration voltages of 10 and $100 \mathrm{kV}$. Each dot is an average of grouped data points (for logarithmic distributed bins) with error bars given by \pm 1 standard deviation (systematic errors have not been included). The measured data points for the different lens positions have been combined into a single data set by plotting $\sigma_{0}$ versus $F_{0}$ instead of $Q$ versus $E$. The data points for the different lens positions connect very well, although some small shifts are noticeable. By moving the lens, the alignment on the cathode might have changed slightly, which might influence the photoemission yield due to

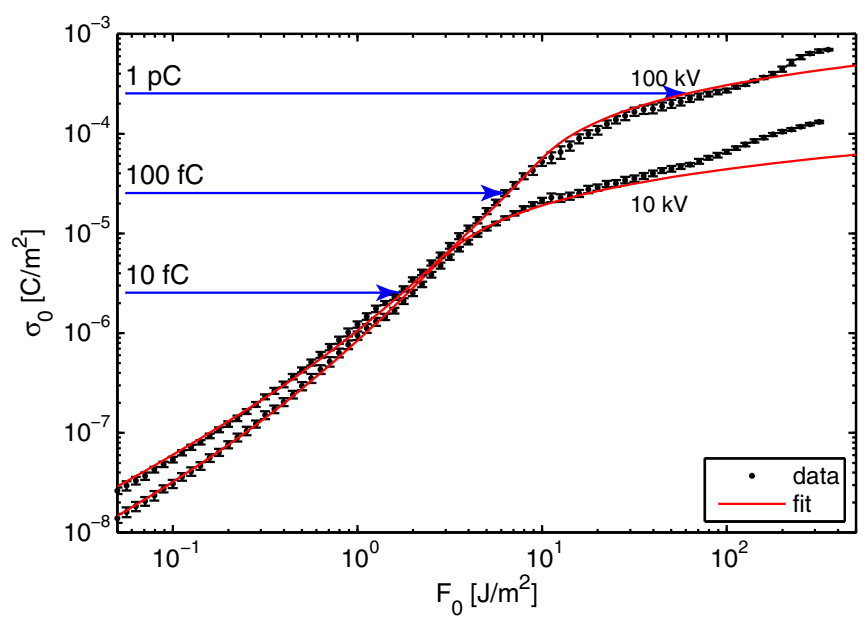

FIG. 3. Measured peak surface charge density $\sigma_{0} \equiv Q /\left(2 \pi s^{2}\right)$ as a function of peak fluence $F_{0}$ for acceleration voltages of 10 and $100 \mathrm{kV}$, corresponding to acceleration fields $\left(G_{a}\right)$ of 1.23 and 12.3 MV/m. The blue arrows indicate the conditions at which waist scans have been performed (at $100 \mathrm{kV}$ ). 
variations in the laser beam profile or due to local variations like surface roughness or quantum efficiency [26].

The damage threshold of copper is on the order of $1 \mathrm{PW} / \mathrm{m}^{2}$ for sub-ps pulses [22]. This is equivalent to a fluence on the order of $100 \mathrm{~J} / \mathrm{m}^{2}$ for a pulse length of $100 \mathrm{fs}$. From this fluence an increase in slope is visible in Fig. 3, which thus seems to be an indication of cathode damaging.

The data have been fitted up to $F_{0}=50 \mathrm{~J} / \mathrm{m}^{2}$ using Eq. (9) for each acceleration voltage, with fitting parameters $b_{1}$ and $b_{2}$, and the assumption $\beta=1$ to calculate $F_{\text {lim }}$ from $b_{1}$ and $b_{2}$. The fitted curves for 10 and $100 \mathrm{kV}$ are shown in Fig. 3 and demonstrate that the measured data agree well with the theory. Similar curves have been recorded and fitted equally well with theory for acceleration voltages $20,30, \ldots, 80,90 \mathrm{kV}$. For $100 \mathrm{kV}$ acceleration voltage, corresponding to $G_{a}=12.3 \mathrm{MV} / \mathrm{m}$, we find $b_{1}=$ $5.4 \times 10^{-7} \mathrm{C} / \mathrm{J}$ and $b_{2}=5.4 \times 10^{-7} \mathrm{Cm}^{2} / \mathrm{J}^{2}$. This means that at a peak fluence $F_{0}=0.1 \mathrm{~J} / \mathrm{m}^{2} 80 \%$ of the electron yield is due to a linear photoemission process, while at $F_{0}=10 \mathrm{~J} / \mathrm{m}^{2} 95 \%$ of the electron yield can be attributed to second order photoemission. The fitted parameters $b_{1}$ and $b_{2}$ and their dependence on acceleration field are discussed in more detail in the following two sections.

\section{B. Schottky effect}

The fitted parameter $b_{1}$ is shown in Fig. 4 as a function of the acceleration field $G_{a}$. The error bars are based on 95\% confidence bounds for the fit and do not include systematic errors. The relatively large error margins are a result of the correlation with $b_{2}$ (see Eq. (9)) and therefore indicate the range of possible values of $b_{1}$. An increase of $b_{1}$ as a function of $G_{a}$ is observed due to the Schottky effect. The data have been fitted using the first-order coefficient of Eq. (7) with the work function $\phi$ from Eq. (1), assuming $h \nu=4.65 \mathrm{eV}$ and $\beta=1$. The values obtained from the fit (with confidence bounds from the fit only) are $\phi_{0}=4.48 \pm 0.01 \mathrm{eV}$ for the zero field work

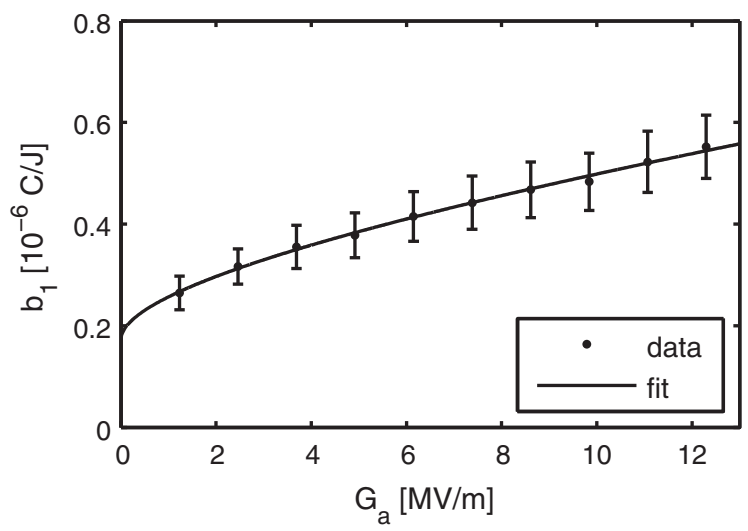

FIG. 4. First-order coefficient $b_{1}$ as a function of acceleration field $G_{a}$. function and $a_{1}=(3.4 \pm 0.4) \times 10^{-19} \mathrm{~m}^{2} / \mathrm{A}$. The value for $\phi_{0}$ fits well within the range $\phi_{0}=4.31-4.91 \mathrm{eV}$ found in literature $[27,28]$. The value for $a_{1}$ is more than an order of magnitude below the value of $5 \times 10^{-18} \mathrm{~m}^{2} / \mathrm{A}$ for atomically clean copper derived from [29] (see Sec. II B). Surface contamination could explain the lower value, especially in view of the fact that the cathode has been in use for 9 months (see also Sec. IV E) and was not cleaned prior to the measurement; the only form of cleaning is the illumination with the UV light pulses.

\section{Second-order photoemission}

The fitted parameter $b_{2}$ is shown in Fig. 5 as a function of the acceleration field $G_{a}$. The error bars are based on 95\% confidence bounds for the fit and do not include systematic errors. The second-order coefficient $b_{2}$ can be explained by thermally assisted one-photon photoemission (1PPE) or by above-threshold two-photon photoemission (2PPE). The case of 1PPE can be described with the second-order coefficient of Eq. (7), which does not depend on $G_{a}$. With $a_{1}$ from the previous section, the mean value of $b_{2}$ gives $\alpha / \kappa=(7.2 \pm 0.2) \times 10^{6} \mathrm{~m}^{2} \mathrm{~K}^{2} / \mathrm{J}$, which is an order of magnitude larger than the value $\alpha / \kappa=$ $8 \times 10^{5} \mathrm{~m}^{2} \mathrm{~K}^{2} / \mathrm{J}$ obtained from literature (see Sec. II C). The case of 2PPE can be described using Eq. (8), with the same parameters as in the previous section combined with the fitted value for $\phi_{0}$. Assuming $\tau=100 \mathrm{fs}, a_{2}$ has been used as a fit parameter. The fitted value $a_{2}=(4.1 \pm 0.2) \times$ $10^{-33} \mathrm{~m}^{4} / \mathrm{A}^{2}$, which agrees well with the value of $a_{2} \approx$ $5 \times 10^{-33} \mathrm{~m}^{4} / \mathrm{A}^{2}$ as estimated in Sec. II C. It is not possible to determine from the experimental data whether the 1PPE or the 2PPE mechanism determines the second-order slope. However, in case of 2PPE, the fitted value $b_{2}$ can be explained well with a value for $a_{2}$ which is in line with literature values, whereas in case of 1PPE the fitted value would have to be explained with a value for $\alpha / \kappa$ which is $\sim 10 \times$ larger than literature values. This could be an indication that $2 \mathrm{PPE}$ is the dominant process causing the

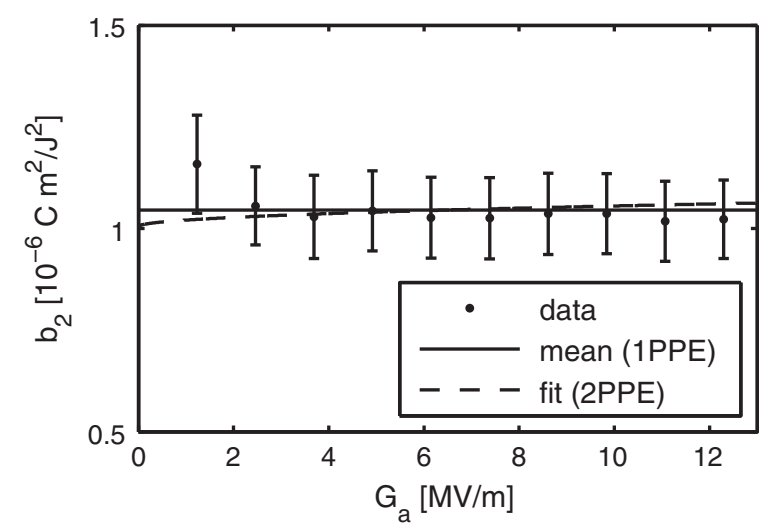

FIG. 5. Second-order coefficient $b_{2}$ as a function of acceleration field $G_{a}$. 
second order photoemission. This is supported by additional measurements on a new cathode, which will be presented in Sec. IV E.

\section{Image charge limited emission}

The image charge limited emission becomes apparent in Fig. 3 at a fluence $F_{a} \approx 10 \mathrm{~J} / \mathrm{m}^{2}$. The influence of the acceleration field is also clear. The data agree well with the model from Sec. II D for $\beta=1$. For $100 \mathrm{kV}$, the fitted curve in Fig. 3 is slightly above the measured data points. This small discrepancy would increase with increasing $\beta$. The measurements thus indicate that $\beta$ should be of order unity, which agrees well with $\beta=1.5 \pm 0.2$ for an $\mathrm{rf}$ photogun reported in Ref. [23], and is in disagreement with values up to 500 reported in Ref. [26].

\section{E. New cathode}

The cathode from which the above results were obtained, had been in use for 9 months at the time of the measurements. After replacing the cathode and training of the dc gun, a single yield measurement has been performed with the new cathode for an acceleration voltage of $100 \mathrm{kV}$ and a spot size $s=90.3 \pm 0.5 \mu \mathrm{m}$. For this measurement another Faraday cup has been used which was mounted in the sample chamber of the UED setup. The Faraday cup consists of a $20 \times 20 \mathrm{~mm}^{2}$ block of copper with a thickness of $8 \mathrm{~mm}$. Due to the back scattered fraction of the electrons at $100 \mathrm{keV}$, the charge measured with this Faraday cup may be somewhat lower, maximally $30 \%$, than if the same had been used as described in Sec. III B. The charge amplifier and the UV diode are identical. The result of this measurement is shown in Fig. 6, along with the measurement at $100 \mathrm{kV}$ for the other cathode. The data has been fitted using Eq. (9) with the assumption $\beta=1$. Note that it is also

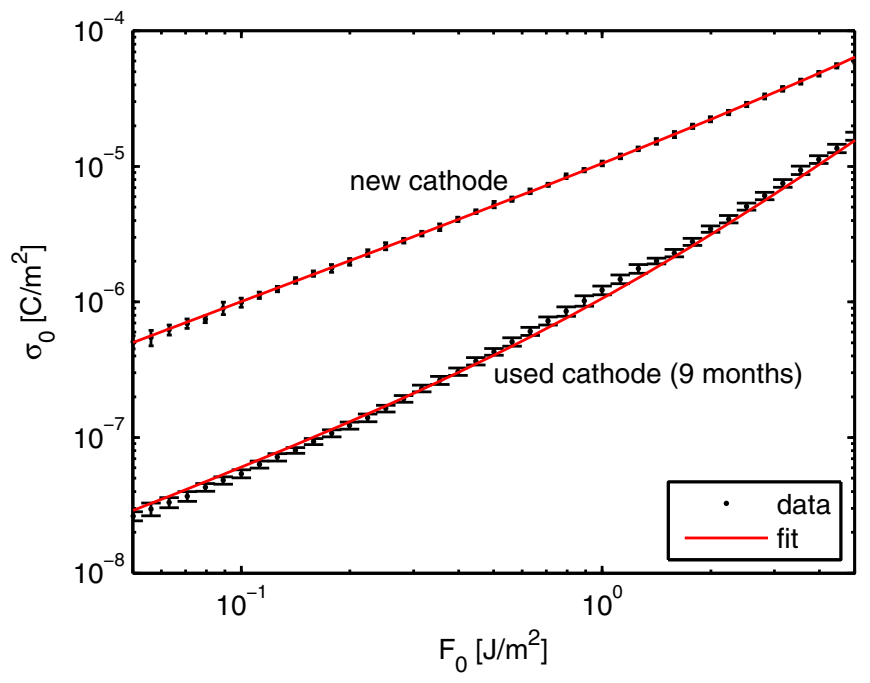

FIG. 6. Measured photoemitted yield at an acceleration voltage of $100 \mathrm{kV}$ for the new and used cathodes.
TABLE II. Photoemission coefficients for the new and used cathodes.

\begin{tabular}{lcc}
\hline \hline & $b_{1}[\mathrm{C} / \mathrm{J}]$ & $b_{2}\left[\mathrm{Cm}^{2} / \mathrm{J}^{2}\right]$ \\
\hline new cathode & $(1.00 \pm 0.01) \times 10^{-5}$ & $(1.13 \pm 0.07) \times 10^{-6}$ \\
used cathode & $(5.5 \pm 0.5) \times 10^{-7}$ & $(1.02 \pm 0.07) \times 10^{-6}$ \\
\hline \hline
\end{tabular}

assumed that the reflectivity of the cathode has remained constant. The fitted coefficients are shown in Table II (with 95\% confidence bounds from the fit).

The value of $b_{1}$ for the new cathode agrees very well with the estimated $b_{1}=1.1 \times 10^{-5} \mathrm{C} / \mathrm{J}$ from Sec. II C. Apparently, the aging effect of the cathode is quite significant, which is also known from literature [25]. Interestingly, the value of $b_{2}$ is almost similar for the new and used cathode. This implies that the electron yield of the new cathode is almost entirely due to a linear photoemission process over the entire range of fluences shown in Fig. 6, in contrast to the used cathode. The fact that the measured charge may have been slightly underestimated due to the use of a Faraday cup with a simpler construction does not change these conclusions. In the case of 1PPE this would imply that $a_{1} \alpha / \kappa$ is constant for both cathodes and in the case of 2PPE that $a_{2}$ is constant. For constant $a_{1}$, the increase of $b_{1}$ could only be explained by assuming that the work function has decreased to a value $\phi_{0}=3.49 \mathrm{eV}$ for the new cathode, which is far below literature values and therefore not very likely. A better explanation is that $a_{1}$ has increased to a value of $6 \times 10^{-18} \mathrm{~m}^{2} / \mathrm{A}$ for the new cathode, in good agreement with the value of $5 \times 10^{-18} \mathrm{~m}^{2} /$ A for atomically clean copper derived from [29]. In the case of 1PPE, however, the increase of $a_{1}$ should also result in an increase of $b_{2}$ with constant $\alpha / \kappa$ for both cathodes. The fact that the value of $b_{2}$ remains the same is therefore an indication that the second-order photoemission is mainly the result of $2 \mathrm{PPE}$.

\section{F. Emittance and brightness}

Figure 7 shows waist scans performed for $100 \mathrm{keV}$ electron bunches with bunch charges of $10 \mathrm{fC}, 100 \mathrm{fC}$, and $1 \mathrm{pC}$ created from a laser spot on the cathode with an rms size $s=25 \mu \mathrm{m}$. The conditions at which these waist scans have been performed are also indicated by the arrows in Fig. 3. Each data point in Fig. 7 has been obtained from five bunches imaged onto the detector (with one image per bunch). Examples of these images are shown in Fig. 8. For each image the background is subtracted and the beam center is determined from integrated intensity profiles, along both the horizontal and vertical direction, which are fitted with a Gaussian function. The pixel intensity is plotted as a function of the distance to the beam center and fitted with a Gaussian function, which provides the rms beam width. The analysis assumes a cylindrically symmetric Gaussian electron distribution. This assumption 


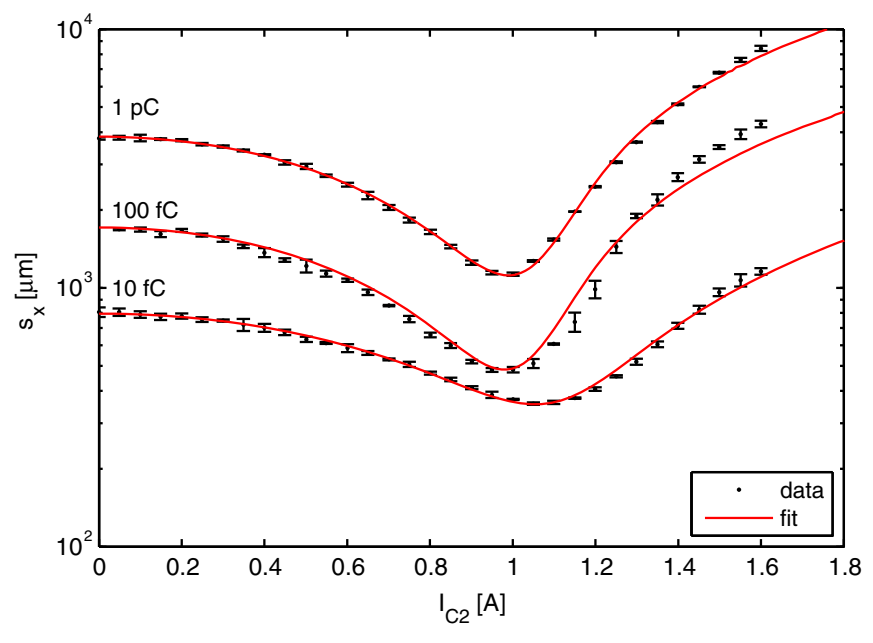

FIG. 7. Waist scans for different bunch charges.

is valid for $10 \mathrm{fC}$ bunches, but for $100 \mathrm{fC}$ and especially for $1 \mathrm{pC}$ bunches the distribution becomes less Gaussian, less symmetric, and less smooth, as is visible in Fig. 8. Although the distribution becomes distorted due to space-charge effects and image charge forces during photoemission, the analysis method nevertheless provides a reasonable rms beam width.

Equation (13) is used to describe the space-charge dominated beam propagation with the generalized perveance $K$ as a fit parameter. A Gaussian beam is assumed described by a transverse geometrical emittance $\epsilon_{x}$ and a virtual source size $s_{0}$ at position $z_{o}$, which are all used as fit parameters. Details on the modeling of the magnetic lens strength as a function of current $I_{C 2}$ can be found in Ref. [42]. The fitted curves are shown in Fig. 7. The fitted
TABLE III. Fitted beam parameters.

\begin{tabular}{lcccc}
\hline \hline$Q$ & $\epsilon_{x}[\mathrm{~nm}]$ & $s_{0}[\mu \mathrm{m}]$ & $z_{o}[\mathrm{~m}]$ & $\mathrm{K}[-]$ \\
\hline $10 \mathrm{fC}$ & $(39 \pm 2)$ & $(159 \pm 2)$ & $(-0.67 \pm 0.05)$ & $(8.1 \pm 0.6) \times 10^{-8}$ \\
$100 \mathrm{fC}$ & $(41 \pm 3)$ & $(145 \pm 12)$ & $(-2.63 \pm 0.25)$ & $(5.1 \pm 0.2) \times 10^{-7}$ \\
$1 \mathrm{pC}$ & $(198 \pm 15)$ & $(303 \pm 18)$ & $(-2.52 \pm 0.09)$ & $(1.9 \pm 0.1) \times 10^{-6}$ \\
\hline \hline
\end{tabular}

parameters, with $95 \%$ confidence bounds from the fitting routine, are listed in Table III.

The initial beam parameters are compared with theoretical parameters for $0 \mathrm{fC}$ (i.e., without space charge) calculated with parameters obtained from particle tracking simulations with GPT [37]. The GPT simulations are performed for the gun and the first magnetic coil using 10000 macroparticles with space charge included using the mesh-based 3D space routine in GPT (except for $0 \mathrm{fC}$, where no space charge is included). For both the calculations and the simulations, a Gaussian beam at the cathode with $k_{B} T_{b}=0.5 \mathrm{eV}, s=25 \mu \mathrm{m}$, and $\tau=100 \mathrm{fs}$ is assumed. The parameters $\epsilon_{x}, s_{0}$, and $z_{o}$ are determined at the position of the second condenser lens and are presented in Table IV. The slight difference in the parameters $s_{0}$ and $z_{0}$ between the theoretical and simulated parameters for $0 \mathrm{fC}$ can be attributed to the fact that the field from the first magnetic coil is not negligible inside the gun, which is assumed for the theoretical beam model. The difference between the parameters obtained with and without Coulomb interactions clearly shows the effect of space charge on emittance and beam divergence.

Comparing Tables III with IV, the parameters are in very good agreement for $10 \mathrm{fC}$. For $100 \mathrm{fC}$ and $1 \mathrm{pC}$, the model and the GPT simulations start to deviate; it is interesting to note that the fitted emittances are actually lower than the
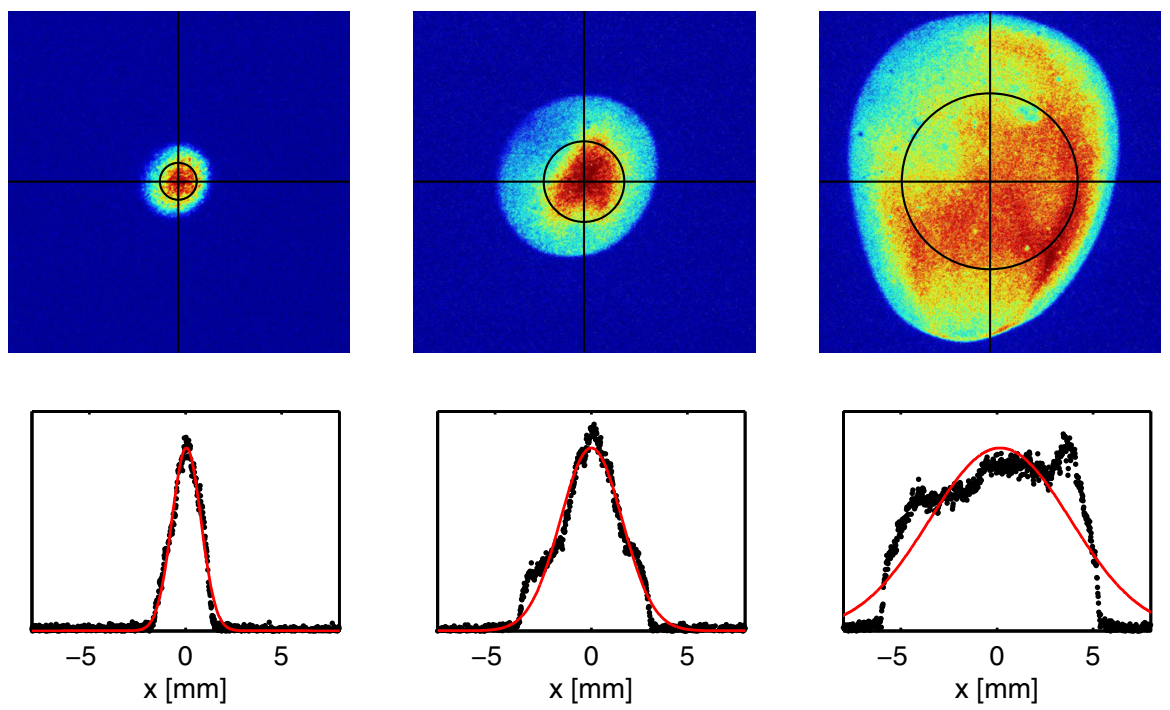

FIG. 8. Top row: images of electron bunches for $I_{C 2}=0.1 \mathrm{~A}$ for $10 \mathrm{fC}, 100 \mathrm{fC}$, and $1 \mathrm{pC}$ (from left to right); the black lines indicate the fitted beam center and rms beam width. Bottom row: horizontal lineouts (black curves) averaged over 40 pixels in the vertical direction; the lineouts are fitted with Gaussian functions shown by the red curves. 
TABLE IV. Beam parameters from theory/GPT.

\begin{tabular}{lccrcr}
\hline \hline$Q$ & $\epsilon_{x}[\mathrm{~nm}]$ & $s_{0}[\mu \mathrm{m}]$ & $z_{o}[\mathrm{~m}]$ & $\tau[\mathrm{ps}]$ & $\mathrm{K}[-]$ \\
\hline 0 fC (theory) & 37.7 & 194 & 0.542 & 0.13 & \\
0 fC (GPT) & 37.7 & 203 & 0.412 & 0.14 & \\
$10 \mathrm{fC}$ & 40.5 & 168 & -0.806 & 8 & $1 \times 10^{-7}$ \\
$100 \mathrm{fC}$ & 70.7 & 130 & -1.032 & 19 & $6 \times 10^{-7}$ \\
$1 \mathrm{pC}$ & 285 & 273 & -1.312 & 29 & $4 \times 10^{-6}$ \\
\hline \hline
\end{tabular}

emittances obtained from GPT simulations. One possible explanation is that the nonlinear photoemission process, which is not included in the GPT simulations, may give rise to a lower emittance. Moreover, the real beam profiles, as shown in Fig. 7, are somewhat asymmetric, especially for $1 \mathrm{pC}$, which will influence the space-charge dynamics during propagation. A detailed analysis of the waist scan data with GPT would require using initial simulation conditions based on the measured laser beam profile, in combination with a model for the nonlinear photoemission process. This is outside the scope of this paper. It is instructive, however, to make estimates for the perveance $K$ using GPT and see whether the $K$ values obtained from the fits make sense. Table IV gives the values of the bunch length $\tau$ at the detector from the GPT simulations and the values of $K$ using $I_{b} \simeq Q / 4 \tau$ ( $\approx 63 \%$ of the peak current) as an estimate for the current. We find that the order of magnitude of the estimated values for $K=I_{b} / I_{P}$ is in agreement with the fitted values.

The $100 \mathrm{fC}$ bunches with a normalized emittance of $25 \mathrm{~nm}$ rad obtained from the waist scans analysis, have the same transverse normalized brightness [Eq. (11)] as $160 \mathrm{pC}$ bunches with a normalized emittance of $1 \mu \mathrm{m} \mathrm{rad}$, which corresponds to a brightness usually associated with $\mathrm{rf}$ photoguns. By optimizing the laser beam profile spacecharge effects may be reduced, which would result in an even higher brightness.

\section{CONCLUSIONS AND OUTLOOK}

Photoemission yield and emittance measurements have been reported for the dc electron gun of the UED setup at Eindhoven University of Technology. The photoemission yield has been studied over a wide range of laser fluence, from the linear photoemission regime until the onset of image charge limitations and cathode damaging. The measured curves can be explained well with available theory including the Schottky effect, second-order photoemission, and image charge limitation. A low field enhancement factor of $\beta=1$ works best to fit the data. The second-order photoemission can be explained by thermally assisted one-photon emission (1PPE) and by above-threshold two-photon emission (2PPE). At the time of the experiments, the copper cathode had been in use for 9 months and was not cleaned prior to the measurements. Additional measurements with a fresh cathode suggest that the 2PPE process is dominant. Two photons would make the highly occupied $\mathrm{Cu}$ d-band accessible for photoemission, which could explain the quite high efficiency of this process. The measurements with the new cathode are also an indication of cathode degradation in time. This stresses the importance of measuring the photoemission yield on a day-to-day basis (if bunch charge is an important parameter) and of regularly cleaning or replacing the cathode (if yield is important).

The influence of the initial charge density on the beam emittance has been studied. The emittance measurements of space-charge dominated beams are described well by an envelope equation with generalized perveance. We find that the transverse brightness obtained with a $12 \mathrm{MV} / \mathrm{m}$, $100 \mathrm{keV}$ dc photogun is comparable to the brightness of $\mathrm{rf}$ photoguns.

We have explored the limits of femtosecond photoemission in a dc gun and understand the underlying mechanisms. It is found that for applications such as UED and UEM, the femtosecond photoemission process will be nonlinear under most relevant conditions. To investigate to what extent the beam quality could further be improved, the second order photoemission process and its dependence on the band structure of the cathode material should therefore be studied in greater detail. This could, for example, be achieved through variation of the laser wavelength, pulse length, and profile, in combination with additional measurements of the electron bunch length and energy spread.

\section{ACKNOWLEDGMENTS}

This research is supported by the Dutch Technology Foundation STW, applied science division of NWO and the Technology Program of the Ministry of Economic Affairs. Expert technical assistance by Eddy Rietman, Ad Kemper, Harry van Doorn, and Iman Koole is gratefully acknowledged.

[1] T. Rao and D. H. Dowell, An Engineering Guide to Photoinjectors (CreateSpace, 2013).

[2] F. Stephan and M. Krasilnikov, in Synchrotron Light Sources and Free-Electron Lasers (Springer, New York, 2016), p. 561.

[3] T. van Oudheusden, E. F. de Jong, S. B. van der Geer, W. P. E. M. Op 't Root, and O. J. Luiten, Electron source concept for single-shot sub-100 fs electron diffraction in the $100 \mathrm{keV}$ range, J. Appl. Phys. 102, 093501 (2007).

[4] F. Carbone, P. Musumeci, O. J. Luiten, and C. Hebert, A perspective on novel sources of ultrashort electron and X-ray pulses, Chem. Phys. 392, 1 (2012).

[5] F. O. Kirchner, S. Lahme, F. Krausz, and P. Baum, Coherence of femtosecond single electrons exceeds biomolecular dimensions, New J. Phys. 15, 063021 (2013). 
[6] A. H. Zewail and J. M. Thomas, 4D Electron Microscopy (Imperial College Press, London, 2010).

[7] R. M. van der Veen, O.-H. Kwon, A. Tissot, A. Hauser, and A.H. Zewail, Single-nanoparticle phase transitions visualized by four-dimensional electron microscopy, Nat. Chem. 5, 395 (2013).

[8] M. Gao, C. Lu, H. Jean-Ruel, L. C. Liu, A. Marx, K. Onda, S. Koshihara, Y. Nakano, X. Shao, T. Hiramatsu, G. Saito, H. Yamochi, R. R. Cooney, G. Moriena, G. Sciaini, and R. J. D. Miller, Mapping molecular motions leading to charge delocalization with ultrabright electrons, Nature (London) 496, 343 (2013).

[9] M. Hada, K. Pichugin, and G. Sciaini, Ultrafast structural dynamics with table top femtosecond hard X-ray and electron diffraction setups, Eur. Phys. J. Spec. Top. 222, 1093 (2013).

[10] S. Schäfer, W. Liang, and A. H. Zewail, Primary structural dynamics in graphite, New J. Phys. 13, 063030 (2011).

[11] N. Erasmus, M. Eichberger, K. Haupt, I. Boshoff, G. Kassier, R. Birmurske, H. Berger, J. Demsar, and H. Schwoerer, Ultrafast Dynamics of Charge Density Waves in $4 H_{b}-\mathrm{TaSe}_{2}$ Probed by Femtosecond Electron Diffraction, Phys. Rev. Lett. 109, 167402 (2012).

[12] E. Blackburn, J. Chang, M. Hücker, A. T. Holmes, N. B. Christensen, R. Liang, D. A. Bonn, W. N. Hardy, U. Rütt, O. Gutowski, M. v. Zimmermann, E. M. Forgan, and S. M. Hayden, X-Ray Diffraction Observations of a Charge-Density-Wave Order in Superconducting Ortho-II $\mathrm{YBa}_{2} \mathrm{Cu}_{3} \mathrm{O}_{6.54}$ Single Crystals in Zero Magnetic Field, Phys. Rev. Lett. 110, 137004 (2013).

[13] L. Redecke et al., Natively inhibited Trypanosoma brucei Cathepsin B structure determined by using an X-ray laser, Science 339, 227 (2013).

[14] M. M. Seibert et al., Single mimivirus particles intercepted and imaged with an X-ray laser, Nature (London) 470, 78 (2011).

[15] S. P. Weathersby et al., Mega-electron-volt ultrafast electron diffraction at SLAC National Accelerator Laboratory, Rev. Sci. Instrum. 86, 073702 (2015).

[16] X. H. Lu, C. X. Tang, R. K. Li, H. To, G. Andonian, and P. Musumeci, Generation and measurement of velocity bunched ultrashort bunch of pC charge, Phys. Rev. ST Accel. Beams 18, 032802 (2015).

[17] R. H. Fowler, The analysis of photoelectric sensitivity curves for clean metals at various temperatures, Phys. Rev. 38, 45 (1931).

[18] L. A. DuBridge, Theory of the energy distribution of photoelectrons, Phys. Rev. 43, 727 (1933).

[19] J. H. Bechtel, W. Lee Smith, and N. Bloembergen, Twophoton photoemission from metals induced by picosecond laser pulses, Phys. Rev. B 15, 4557 (1977).

[20] Photoemission and the Electronic Properties of Surfaces, edited by B. Feuerbacher, B. Fitton, and R. F. Willis (John Wiley \& Sons, New York, 1978).

[21] M. Peloi, G. Ferrini, G. Banfi, G. Secondi, and F. Parmigiani, Non-linear photoemission from polycrystalline molybdenum irradiated by $790 \mathrm{~nm}-150$ fs laser pulses, Solid State Commun. 118, 339 (2001).

[22] G. Ferrini, F. Banfi, C. Giannetti, and F. Parmigiani, Nonlinear electron photoemission from metals with ultrashort pulses, Nucl. Instrum. Methods Phys. Res., Sect. A 601, 123 (2009).

[23] P. Musumeci, L. Cultrera, M. Ferrario, D. Filippetto, G. Gatti, M. S. Gutierrez, J. T. Moody, N. Moore, J. B. Rosenzweig, C. M. Scoby, G. Travish, and C. Vicario, Multiphoton Photoemission from a Copper Cathode Illuminated by Ultrashort Laser Pulses in an rf Photoinjector, Phys. Rev. Lett. 104, 084801 (2010).

[24] Z. Tao, H. Zhang, P. M. Duxbury, M. Berz, and C.-Y. Ruan, Space charge effects in ultrafast electron diffraction and imaging, J. Appl. Phys. 111, 044316 (2012).

[25] F. Zhou, A. Brachmann, F.-J. Decker, P. Emma, S. Gilevich, R. Iverson, P. Stefan, and J. Turner, Highbrightness electron beam evolution following laser-based cleaning of a photocathode, Phys. Rev. ST Accel. Beams 15, 090703 (2012).

[26] H. Chen, Y. Du, W. Gai, A. Grudiev, J. Hua, W. Huang, J. G. Power, E. E. Wisniewski, W. Wuensch, C. Tang, L. Yan, and Y. You, Surface-Emission Studies in a High-Field RF Gun based on Measurements of Field Emission and Schottky-Enabled Photoemission, Phys. Rev. Lett. 109, 204802 (2012).

[27] P. O. Gartland, S. Berge, and B. J. Slagsvold, Photoelectric Work Function of a Copper Single Crystal for the (100), (110), (111), and (112) Faces, Phys. Rev. Lett. 28, 738 (1972).

[28] D. H. Dowell, F. K. King, R. E. Kirby, J. F. Schmerge, and J. M. Smedley, In situ cleaning of metal cathodes using a hydrogen ion beam, Phys. Rev. ST Accel. Beams 9, 063502 (2006).

[29] D. H. Dowell and J. F. Schmerge, Quantum efficiency and thermal emittance of metal photocathodes, Phys. Rev. ST Accel. Beams 12, 074201 (2009).

[30] K. L. Jensen, N. A. Moody, D. W. Feldman, E. J. Montgomery, and P. G. OShea, Photoemission from metals and cesiated surfaces, J. Appl. Phys. 102, 074902 (2007).

[31] C. R. Crowell, The Richardson constant for thermionic emission in Schottky barrier diodes, Solid State Electron. 8, 395 (1965).

[32] Refractive index database, http://refractiveindex.info.

[33] P. B. Johnson and R. W. Christy, Optical constants of the noble metals, Phys. Rev. B 6, 4370 (1972).

[34] Z. Lin, L. V. Zhigilei, and V. Celli, Electron-phonon coupling and electron heat capacity of metals under conditions of strong electron-phonon nonequilibrium, Phys. Rev. B 77, 075133 (2008).

[35] D. M. Riffe, X. Y. Wang, M. C. Downer, D. L. Fisher, T. Tajima, and J. L. Erskine, Femtosecond thermionic emission from metals in the space-charge-limited regime, J. Opt. Soc. Am. B 10, 1424 (1993).

[36] A. Valfells, D. W. Feldman, M. Virgo, P. G. O'Shea, and Y. Y. Lau, Effects of pulse-length and emitter area on virtual cathode formation in electron guns, Phys. Plasmas 9, 2377 (2002).

[37] The general particle tracer (gpt) code, http://www.pulsar.nl.

[38] O. J. Luiten, B. J. Claessens, S. B. van der Geer, M. P. Reijnders, G. Taban, and E. J. D. Vredenbregt, Ultracold electron sources, Int. J. Mod. Phys. A 22, 3882 (2007).

[39] M. Reiser, Theory and Design of Charged Particle Beams, 2nd ed. (Wiley-VCH, New York, 2008). 
[40] T. van Oudheusden, P. L. E. M. Pasmans, S. B. van der Geer, M. J. de Loos, M. J. van der Wiel, and O.J. Luiten, Compression of Subrelativistic Space-ChargeDominated Electron Bunches for Single-Shot Femtosecond Electron Diffraction, Phys. Rev. Lett. 105, 264801 (2010).
[41] T. van Oudheusden, Ph.D. thesis, Eindhoven University of Technology, 2010.

[42] P. L. E. M. Pasmans, Ph.D. thesis, Eindhoven University of Technology, 2014.

[43] F. B. Kiewiet, Ph.D. thesis, Eindhoven University of Technology, 2003. 